Bull. Korean Math. Soc. 39 (2002), No. 1, pp. 81-87

\title{
HARDY'S INEQUALITY RELATED TO A BERNOULLI EQUATION
}

\author{
Jung-Soon Hyun and SAng Dong Kim
}

\begin{abstract}
The weighted Hardy's inequality is known as

$$
\int_{a}^{b}|u(x)|^{p} r(x) d x \leq C \int_{a}^{b}\left|u^{\prime}(x)\right|^{p} s(x) d x
$$

where $-\infty \leq a \leq b \leq \infty$ and $1<p<\infty$. The purpose of this article is to provide a useful formula to express the weight $r(x)$ in terms of $s(x)$ or vice versa employing a Bernoulli equation having the other weight as coefficients.
\end{abstract}

\section{Introduction}

The classical Hardy's inequality [7] states that for $1<p<\infty$ and $\epsilon \neq p-1$

$$
\int_{0}^{\infty}|u(x)|^{p} x^{\epsilon-p} d x \leq\left(\frac{p}{|\epsilon-p+1|}\right)^{p} \int_{0}^{\infty}\left|u^{\prime}(x)\right|^{p} x^{\epsilon} d x
$$

provided $u(0)=0$ for $\epsilon<p-1$ and $u(\infty)=0$ for $\epsilon>p-1$. For singular value $\epsilon=p-1$ for the Hardy's inequality (1.1), Kadlec and Kufner [6] showed

$$
\int_{0}^{1}|u(x)|^{p} \frac{1}{x}|\log x|^{-p} d x \leq C_{p} \int_{0}^{1}\left|u^{\prime}(x)\right|^{p} x^{p-1} d x .
$$

This inequality asks for which weights $r(x)$ and $s(x)$ the following inequality

$$
\int_{a}^{b}|u(x)|^{p} r(x) d x \leq C \int_{a}^{b}\left|u^{\prime}(x)\right|^{p} s(x) d x
$$

Received March 22, 2001.

2000 Mathematics Subject Classification: 26D10.

Key words and phrases: Hardy's inequality, Bernoulli equation.

Partially supported by Brain Korea 21 and KOSEF under grant number 1999-2103-002-3. 
holds. For this question, Muckenhoupt [8] showed that the formula (1.3) holds if and only if

$$
\sup _{0 \leq t \leq \infty}\left(\int_{t}^{\infty}|r(x)|^{p} d x\right)^{1 / p}\left(\int_{0}^{t}|s(x)|^{q} d x\right)^{1 / q}=K<\infty
$$

and $K \leq C \leq K p^{\frac{1}{p}} q^{\frac{1}{q}}$ where $\frac{1}{p}+\frac{1}{q}=1$. In a more general setting, Gurka [5] gave a necessary and sufficient condition for the weights $r(x)$ and $s(x)$, for which

$$
\int_{0}^{\infty}|u(x)|^{p} r(x) d x \leq C \int_{0}^{\infty}\left|u^{\prime}(x)\right|^{q} s(x) d x
$$

holds for $1<p \leq q<\infty$. Kufner and Triebel [7] also gave explicit formulae for $r(x)$ and $s(x)$ for the case $p=q$. In this article, we derive a simple formula such as

$$
r(x)=s(x)^{-\frac{1}{p-1}}\left(c+\frac{1}{K(p-1)} \int s(x)^{-\frac{1}{p-1}} d x\right)^{-p},
$$

which is the solution of a Bernoulli equation (see (2.4)), to find one weight function for a given other weight function. An application of such a formula can be used in a process showing coercivity of variational formulation arisen in spectral method (see [1], [4]) in an area of numerical partial differential equations. For this purpose, we use the formula above with Jacobi weights $s(x)=(1-x)^{a}(1-x)^{b},(a, b>-1)$ to set Hardy's inequality with corresponding weights, $r(x)$ in this last section. For $-1<a, b<1$, the Hardy's inequality is found in [3, p. 91] for example.

\section{Hardy's inequality and a Bernoulli equation}

In this section, we relate two weights $r(x)$ and $s(x)$ in the weighted Hardy's inequality (1.3) in terms of a Bernoulli equation, that is, one weight is a solution of a Bernoulli equation with coefficients as other weight.

Proposition 2.1. Let $1<p<\infty$ and $-\infty \leq a \leq b \leq \infty$. Suppose $r(x)>0$ and $s(x)>0$. Assume

$$
\int_{a}^{b}|f(x)|^{p} s(x) d x<\infty
$$


Then

(2.1) $\left(\int_{a}^{b}\left(\int_{a}^{x}|f(t)| d t\right)^{p} r(x) d x\right)^{1 / p} \leq K p\left(\int_{a}^{b}|f(x)|^{p} s(x) d x\right)^{1 / p}$

holds if

$$
r(x)^{-\frac{p-1}{p}} s(x)^{-\frac{1}{p}} \int_{x}^{b} r(t) d t=K<\infty,
$$

where $K$ is a constant.

Proof. For each $\epsilon>0$ define

$$
F_{\epsilon}(x)=\int_{a}^{x}\left|f_{\epsilon}(t)\right| d t
$$

where $f_{\epsilon}(t)=f(t)$ for $a+\epsilon \leq x \leq b-\epsilon$ and 0 otherwise. Since

$$
F_{\epsilon}(x)^{p}=\int_{a}^{x}\left(F_{\epsilon}(t)^{p}\right)^{\prime} d t=p \int_{a}^{x} F_{\epsilon}(t)^{p-1}\left|f_{\epsilon}(t)\right| d t,
$$

by using Fubini's theorem and Hölder's inequality and (2.2) we have

$$
\begin{aligned}
& \int_{a}^{b} F_{\epsilon}(x)^{p} r(x) d x \\
= & p \int_{a}^{b} \int_{a}^{x} F_{\epsilon}(t)^{p-1}\left|f_{\epsilon}(t)\right| r(x) d t d x \\
= & p \int_{a}^{b} F_{\epsilon}(x)^{p-1}\left|f_{\epsilon}(x)\right| \int_{x}^{b} r(t) d t d x \\
= & p \int_{a}^{b}\left[\left(F_{\epsilon}(x) r(x)^{\frac{1}{p}}\right)^{p-1}\left(\left|f_{\epsilon}(x)\right| s(x)^{\frac{1}{p}}\right) r(x)^{-\frac{p-1}{p}} s(x)^{-\frac{1}{p}} \int_{x}^{b} r(t) d t\right] d x \\
\leq & K p\left(\int_{a}^{b} F_{\epsilon}(x)^{p} r(x)\right)^{\frac{1}{q}}\left(\int_{a}^{b}\left|f_{\epsilon}(x)\right|^{p} s(x) d x\right)^{\frac{1}{p}}
\end{aligned}
$$

where $\frac{1}{p}+\frac{1}{q}=1$. Hence this implies

$$
\left(\int_{a}^{b} F_{\epsilon}(x)^{p} r(x) d x\right)^{\frac{1}{p}} \leq K p\left(\int_{a}^{b}\left|f_{\epsilon}(x)\right|^{p} s(x) d x\right)^{\frac{1}{p}} .
$$

Finally, applying the Dominated Convergence Theorem yields (2.1).

From the relation (2.2), it is obvious to find $s(x)$ for given $r(x)$ and $K$. Conversely if $s(x)$ and $K$ are given, we can find $r(x)$ in the following way: 
Theorem 2.2. For a given weight $s(x)$ and a constant $K$, if $r(x)$ satisfy (2.2), then $r(x)$ is given by

$$
r(x)=s(x)^{-\frac{1}{p-1}}\left(c+\frac{1}{K(p-1)} \int s(x)^{-\frac{1}{p-1}} d x\right)^{-p}
$$

which is a solution of a Bernoulli equation,

$$
r^{\prime}(x)+\frac{1}{p-1}(\log s(x))^{\prime} r(x)=-\frac{p}{p-1} \frac{1}{K} s(x)^{-\frac{1}{p}} r(x)^{1+\frac{1}{p}}
$$

where $c$ is an arbitrary constant.

Proof. By differentiating (2.2), we have a nonlinear equation (2.4) which is of Bernoulli equation and it is well known that the general solution is given by $(2.3)$.

The formula (2.3) provides the way to get other weight for a given weight in the Hardy's inequality (1.3). As applications of the above theorem, we present two corollaries, which imply the classical case (1.1) and the singular case (1.2) by choosing particular weight $s(x)$ in the formula (2.3).

Corollary 2.3. Let $1<p<\infty$, and $\epsilon \neq p-1$. Let $u(x)$ be a function differentiable almost everywhere on $(0, \infty)$ and such that

$$
\int_{0}^{\infty}\left|u^{\prime}(x)\right|^{p} x^{\epsilon} d x<\infty
$$

Moreover, let $u(0)=0$ for $\epsilon<p-1$ and $u(\infty)=0$ for $\epsilon>p-1$. If $s(x)=x^{\epsilon}$, then the classical inequality (1.1) holds.

Proof. Consider the case $\epsilon<p-1$. By choosing $f(x)=u^{\prime}(x)$ in $(2.1)$ and $c=0$ in $(2.3), r(x)$ is given by

$$
\left(\frac{1}{K(p-1-\epsilon)}\right)^{-p} x^{\epsilon-p}
$$

Putting $r(x)$ into (2.1) we have

$$
\left(\int_{0}^{\infty}|u(x)|^{p} x^{\epsilon-p} d x\right)^{\frac{1}{p}} \leq \frac{p}{p-1-\epsilon}\left(\int_{0}^{\infty}\left|u^{\prime}(x)\right|^{p} x^{\epsilon} d x\right)^{\frac{1}{p}} .
$$

For the case $\epsilon>p-1$, the same argument works with $F_{\epsilon}(x)=\int_{x}^{b}\left|f_{\epsilon}(t)\right| d t$. Hence, combining these two cases yields (1.1).

CoROLLARY 2.4. Let $1<p<\infty$, and $u(0)=0$. If $s(x)=x^{p-1}$, then the classical inequality for the singular case (1.2) holds for $a=0$ and $b=1$. 
Proof. For $s(x)=x^{p-1}, r(x)$ in (2.3) is now $\left(K \frac{1}{p-1}\right)^{-p} \frac{1}{x}|\log x|^{-p}$, which is a singular case (1.2).

\section{Hardy's inequalities with Jacobi weights}

In this section, we take Jacobi weights $w(x)=(1-x)^{a}(1+x)^{b},(a, b>$ -1) as an application due to the concise formula (2.3) for the corresponding Hardy's inequalities used in spectral method, which is one of very accurate methods to approximate a solution for partial differential equation numerically, (see $[1,4]$ ). For $-1<a, b<1$, the following result (3.2) coincides with that in $[3$, p.91] and $[2$, p. 378] for the first kind Chebyshev weight $a=b=-\frac{1}{2}$, but we present Hardy's inequality for a general Jacobi weights $(-1<a, b<\infty)$ in the following theorem using a Bernoulli equation (2.4). Let $p=2$ and $s(x)=(1-x)^{a}$ on an interval $(-1,1)$. By Theorem 2.2 when $c=0, r(x)$ is given by

$$
r(x)=\left\{\begin{array}{l}
(K(1-a))^{2}(1-x)^{a-2},-1<a<1, a>1, \\
K^{2} \frac{1}{1-x}(\log (1-x))^{-2}, a=1,
\end{array}\right.
$$

and we may have, for $s(x)=(1+x)^{b}$,

$$
r(x)=\left\{\begin{array}{l}
(K(1-a))^{2}(1+x)^{b-2},-1<b<1, b>1, \\
K^{2} \frac{1}{1+x}(\log (1+x))^{-2} b=1 .
\end{array}\right.
$$

Note that for such pairs of $r(x)$ and $s(x),(2.2)$ is satisfied. Define a weight $w_{1}(x)=r_{a}(x) r_{b}(x)$ where

$$
r_{a}(x)=\left\{\begin{array}{l}
(1-x)^{a-2},-1<a<1, a>1, \\
\frac{1}{1-x}(\log (1-x))^{-2}, a=1,
\end{array}\right.
$$

and

$$
r_{b}(x)=\left\{\begin{array}{l}
(1+x)^{b-2},-1<b<1, b>1, \\
\frac{1}{1+x}(\log (1+x))^{-2} b=1 .
\end{array}\right.
$$

Let $H_{w, o}^{1}=\left\{\left.u \in L^{2}(-1,1)\left|\int_{-1}^{1} u(x)^{2} w(x) d x<\infty, \int_{-1}^{1}\right| u^{\prime}(x)\right|^{2} w(x) d x<\right.$ $\infty, u(-1)=u(1)=0\}$. 
Theorem 3.1. Let $w_{2}(x)=(1-x)^{a}(1+x)^{b}$ where $a>-1$ and $b>-1$. Then for all $u \in H_{w_{2}, o}^{1}$ we have the following Hardy's inequality

$$
\int_{-1}^{1} u(x)^{2} w_{1}(x) d x \leq C \int_{-1}^{1} u^{\prime}(x)^{2} w_{2}(x) d x
$$

where $w_{1}(x)$ is defined above with suitable choice depending on $a$ and $b$, and $C=4 \max \left\{m_{a}^{-1} M_{a}(1-b)^{-2}, m_{b}^{-1} M_{b}(1-a)^{-2}\right\}$ for $a, b \neq 1$ and the constant $C$ will not contain the term $(1-a)^{-2}$ or $(1-b)^{-2}$ for the case $a=1$ or $b=1$. The constants $m_{a}, M_{a}, m_{b}$ and $M_{b}$ are defined below.

Proof. For $a, b \neq 1$, applying Proposition 2.1 into two weights $r(x)$ and $s(x)$ in (3.1)

$$
\begin{aligned}
\int_{0}^{1} u(x)^{2} r_{a}(x) d x & =(K(1-a))^{-2} \int_{0}^{1}\left(\int_{x}^{1} u^{\prime}(s) d s\right)^{2} r(x) d x \\
& \leq 4(1-a)^{-2} \int_{0}^{1} u^{\prime}(x)^{2}(1-x)^{a} d x \\
& \leq 4 m_{b}^{-1}(1-a)^{-2} \int_{0}^{1} u^{\prime}(x)^{2}(1-x)^{a}(1+x)^{b} d x
\end{aligned}
$$

since $m_{b}^{-1}(1+x)^{b} \geq 1$ on $[0,1]$ where $m_{b}=\min \left(1,2^{b}\right)$. A similar argument shows

$$
\int_{-1}^{0} u(x)^{2} r_{b}(x) d x \leq 4 m_{a}^{-1}(1-b)^{-2} \int_{-1}^{0} u^{\prime}(x)^{2}(1-x)^{a}(1+x)^{b} d x
$$

so that the result (3.2) now follows from

$$
\int_{-1}^{1} u(x)^{2} r(x) d x \leq M_{a} \int_{-1}^{0} u(x)^{2} r_{b}(x) d x+M_{b} \int_{0}^{1} u(x)^{2} r_{a}(x) d x
$$

where $M_{a}=\max \left(1,2^{a}\right)$ and $M_{b}, m_{a}$ are defined analogously. The proof for the case $a=1$ or $b=1$ is same.

\section{References}

[1] C. Bernardi and Y. Maday, Approximations spectrales de Problèmes aux limites elliptiques, Springer-Verlag, Paris, 1992.

[2] C. Canuto, M. Y. Hussaini. A. Quarteroni, and T. A. Zang, Spectral methods in Fluid Dynamics, Springer-Verlag, 1987.

[3] D. Funaro, Polynomial Approximation of Differential equations, Lecture Notes in Physics, Springer-Verlag, 1992.

[4] D. Gottlieb and S. A. Orszag, Numerical Analysis of Spectral Methods: Theory and Applications, CBMS-NSF Regional Conference in Applied Mathematics, SIAM 26 (1993). 
[5] P. Gurka, Generalized Hardy's Inequality, Casopis Pro. 109 (1984), 194-203.

[6] A. Kadlec and A. Kufner, Characterization of functions with zero traces by integrals with weight functions $I, I I$., Casopis Pest. Mat. 91 (1967), 16-28.

[7] R. A. Kufner and H. Triebel, Generalization of Hardy's inequality, Conf. Sem. Mat. Univ. Bari 156 (1978), 1-21.

[8] B. Muckenhoupt, Hardy's Inequality with weights, Studia Math. 44 (1972), 31-38.

Jung-Soon Hyun, Financial EngineEring Research Center, Graduate School of MANagement KAIST, SEOUl 130-012, Korea

Emmail: jshyun@kgsm.kaist.ac.kr

Sang Dong Kim, Department of Mathematics Education, KyUngrook NaTIONAL UNIVERSITY, TAEGU 702-701, KOREA

E-mail: skim@sobolev.knu.ac.kr 